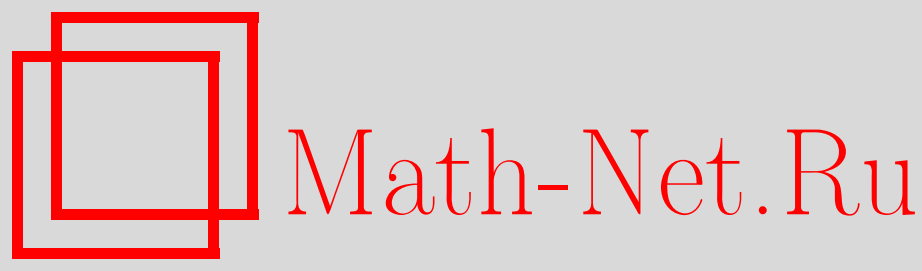

В. С. Герджиков, Г. Г. Граховски, А. В. Михайлов, Т. И. Валчев, Рациональные пучки и рекурсионные операторы для интегрируемых уравнений на симметричных пространствах типа A.III, TMФ, 2011, том 167, номер 3, 394-406

DOI: https://doi.org/10.4213/tmf6649

Использование Общероссийского математического портала Math-Net.Ru подразумевает, что вы прочитали и согласны с пользовательским соглашением http://www . mathnet.ru/rus/agreement

Параметры загрузки:

IP : 3.85 .7 .115

26 апреля 2023 г., 14:22:59

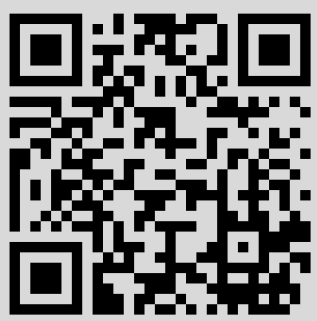




\title{
РАЦИОНАЛЬНЫЕ ПУЧКИ И РЕКУРСИОННЫЕ ОПЕРАТОРЫ ДЛЯ ИНТЕГРИРУЕМЫХ УРАВНЕНИЙ НА СИММЕТРИЧНЫХ ПРОСТРАНСТВАХ ТИПА А.III
}

\begin{abstract}
Проведен анализ и сравнение методов построения рекурсионных операторов для специального класса интегрируемых нелинейных дифференциальных уравнений, связанных с симметричными пространствами типа A.III в классификации Картана и имеющих дополнительные редукции.
\end{abstract}

Ключевые слова: рациональные пучки, интегрируемые уравнения, рекурсионные операторы.

\section{1. ВВЕДЕНИЕ}

Рекурсионные операторы $\Lambda_{ \pm}$играют важную роль в теории нелинейных эволюционных уравнений (НЭУ), интегрируемых с помощью метода обратной задачи рассеяния (МОЗР). Они были построены и проанализированы для широкого класса операторов Лакса $L$, и оказалось, что они порождают не только представления Лакса, но также иерархию НЭУ, связанных с данным оператором Лакса $L$, их законы сохранения и иерархию гамильтоновых структур (см. работы [1]-[4] и многочисленные ссылки в них). Рекурсионный оператор также может рассматриваться как оператор Лакса $L$, взятый в сопряженном представлении соответствующей алгебры Ли g.

Операторы $\Lambda_{ \pm}$для операторов Лакса, явная зависимость которых от спектрального параметра $\lambda$ является сравнительно простой (скажем, линейной или квадратичной), были построены давно [5]-[8]. Кроме того, крайне важным является свойство полноты набора собственных функций операторов $\Lambda_{ \pm}$(“квадратов решений” оператора $L)$. Полнота “квадратов решений” играет фундаментальную роль в доказательстве того, что МОЗР является фактически нелинейным аналогом преобразования

*Institute of Nuclear Research and Nuclear Energy, Bulgarian Academy of Sciences, Sofia, Bulgaria. E-mail: gerjikov@inrne.bas.bg, valtchev@inrne.bas.bg

${ }^{\dagger}$ School of Mathematical Sciences, Dublin Institute of Technology, Dublin, Ireland. E-mail: grah@inrne.bas.bg

${ }^{\ddagger}$ Applied Mathematics Department, University of Leeds, Leeds, UK.

E-mail: a.v.mikhailov@leeds.ac.uk 
Фурье, который позволяет линеаризовать НЭУ. На этом пути можно вывести все фундаментальные свойства НЭУ единым образом.

Важным инструментом выделения новых НЭУ из известных многокомпонентных НЭУ является группа редукций, введенная в работах [9]-[12]. Появление этого метода привело к формулировке теорий двумерного поля Тоды [11], [13], а самые последние достижения состоят в открытии новых автоморфных алгебр Ли и их классификации [14]-[16].

При наличии дополнительных редукций проблема получения рекурсионных операторов становится более сложной. Если редукция совместна с линейным или квадратичным по $\lambda$ оператором $L$, то построение оператора $\Lambda$ является сравнительно простой задачей [4]. Альтернативное построение оператора $\Lambda$ как формального оператора редукции дается в работах [17], [18] (см. также обзоры [19], [20]). В результате $\mathbb{Z}_{n}$-редукции, во-первых, соответствующие "квадраты решений" являются аналитическими в секторах комплексной плоскости переменной $\lambda$, ограниченных углами $\pi / n$; во-вторых, можно воспользоваться градуировкой алгебры Ли $\mathfrak{g} \equiv \bigoplus_{k=0}^{n-1} \mathfrak{g}^{(k)}$, и, как следствие, рекурсионный оператор разлагается в произведение $n$ сомножителей, $\Lambda=\prod_{k=0}^{n-1} \Lambda_{k}$, где каждый из сомножителей $\Lambda_{k}$ осуществляет отображение $\Lambda_{k}: \mathfrak{g}^{(k-1)} \rightarrow \mathfrak{g}^{(k)}$.

Ситуация становится более сложной, если дополнительная редукция радикально изменяет зависимость оператора Лакса от $\lambda$. В настоящей работе мы рассмотрим эту проблему в одном из простейших случаев, когда оператор Лакса $L$ изменяет полиномиальную зависимость от $\lambda$ на рациональную. Как было показано ранее, линейный по $\lambda$ оператор Лакса, задаваемый равенством

$$
L_{0} \psi:=i \partial_{x} \psi+\lambda L_{1} \psi(x, \lambda)=0
$$

приводит к интегрируемой системе [17], [21]

$$
i \boldsymbol{u}_{t}=\left(\left(1-\boldsymbol{u} \boldsymbol{u}^{\dagger}\right) \boldsymbol{u}_{x}\right)_{x}, \quad \boldsymbol{u}^{\dagger} \boldsymbol{u}=\mathbb{1}
$$

где $\boldsymbol{u}$ - комплексная матрица размера $(N-k) \times k$, а $\mathbb{1}$ - единичная матрица размера $k \times k$. Система (1) является $S(U(N-k) \times U(k))$-инвариантной и в этом смысле изотропной. В частности, если $k=1$, то систему (1) можно рассматривать как $U(N-1)$-инвариантную интегрируемую систему в пространстве $\mathbb{C P}^{N-1}$. Соответствующие рекурсионные операторы были выведены в работе [22].

В работе [21] мы получили новые НЭУ с дополнительной $\mathbb{Z}_{2}$-редукцией, которая содержит преобразование $\lambda \rightarrow \lambda^{-1}$ и является одной из простейших нетривиальных редукций (см. [10]-[12], [15], [16]). Благодаря ей соответствующий оператор Лакса приобретает рациональную зависимость от $\lambda$,

$$
L \psi:=i \partial_{x} \psi+\left(\lambda L_{1}+\frac{1}{\lambda} L_{-1}\right) \psi=0,
$$

а простейшие НЭУ принимают вид

$$
\begin{aligned}
i u_{t} & =u_{x x}-\left(u\left(u^{*} u_{x}+v^{*} v_{x}\right)\right)_{x}+8 v v^{*} u, \\
i v_{t} & =v_{x x}-\left(v\left(u^{*} u_{x}+v^{*} v_{x}\right)\right)_{x}-8 u u^{*} v,
\end{aligned}
$$


где $u$ и $v$ - комплексные функции от $x$ и $t$, подчиняющиеся условию $\left|u^{2}\right|+\left|v^{2}\right|=1$, т. е. вектор с компонентами $u, v$ пробегает трехмерную сферу в $\mathbb{R}^{4}$. Система (3) может также рассматриваться как анизотропная деформация уравнения (1) при $k=1$, $N=3$.

Главная цель настоящей работы - предложить два метода получения рекурсионных операторов, связанных с операторами Лакса, имеющими рациональную зависимость от $\lambda$. Результаты настоящей работы являются естественным продолжением наших предыдущих результатов [21], [22] и работы [23]. В разделе 2 мы приводим предварительные сведения, касающиеся спектральных свойств оператора $L$ (2). В разделе 3 мы выводим рекурсионный оператор с использованием метода Гюрсес-Карасу-Соколова (ГКС). В разделе 4 мы используем соотношения Вронского для определения "квадратов решений" оператора $L$. Затем, используя калибровочно-ковариантный подход [4], [6], мы вводим рекурсионный оператор как оператор, для которого “квадраты решений" являются собственными функциями. Подробности наших расчетов приводятся в приложении.

\section{2. ПРЕДВАРИТЕЛЬНЫЕ СВЕДЕНИЯ}

Сформулируем основные результаты нашей предыдущей работы [21]. В ней мы показали, что уравнения (3), которые естественным образом связаны с симметричным пространством $S U(3) / S(U(1) \times U(2))$ типа A.III [24], допускают представление Лакса и могут быть решены с помощью МОЗР. Оператор Лакса задается соотношением (2), а само уравнение является условием совместности оператора $L$ и линейного оператора $A$, задаваемого равенством

$$
A \psi:=i \partial_{t} \psi+\left(A_{0}+\lambda A_{1}+\frac{1}{\lambda} A_{-1}+\lambda^{2} A_{2}+\frac{1}{\lambda^{2}} A_{-2}\right) \psi=\psi f(\lambda)
$$

Хорошо известно, что вышеупомянутое симметричное пространство построено с помощью инволютивного автоморфизма Картана [24], который приводит к $\mathbb{Z}_{2}$-градуировке в основополагающей алгебре Ли: $\mathfrak{g}=\mathfrak{g}^{(0)} \oplus \mathfrak{g}^{(1)}$, где

$$
\begin{gathered}
\mathfrak{g}^{(0)}=\left\{Y \in \mathfrak{g}: J_{1} Y J_{1}=Y\right\}, \quad \mathfrak{g}^{(1)}=\left\{X \in \mathfrak{g}: J_{1} X J_{1}=-X\right\}, \\
J_{1}=\operatorname{diag}(1,-1,-1) .
\end{gathered}
$$

Наше представление Лакса таково, что $L_{ \pm 1}, A_{ \pm 1} \in \mathfrak{g}^{(1)}$ и $A_{0}, A_{ \pm 2} \in \mathfrak{g}^{(0)} ;$ подробности содержатся в приложении. В частности, для операторов $L_{1}$ и $A_{1}$ мы имеем выражения

$$
L_{1}=\left(\begin{array}{ccc}
0 & u & v \\
u^{*} & 0 & 0 \\
v^{*} & 0 & 0
\end{array}\right), \quad A_{1}=\left(\begin{array}{ccc}
0 & a & b \\
a^{*} & 0 & 0 \\
b^{*} & 0 & 0
\end{array}\right)
$$

которые явно влекут два первых условия редукции

$$
L_{ \pm 1}^{\dagger}=L_{ \pm 1}, \quad A_{ \pm 1}^{\dagger}=A_{ \pm 1}, \quad A_{ \pm 2}^{\dagger}=A_{ \pm 2} .
$$

Мы налагаем также третью редукцию в виде

$$
J_{2} L_{1} J_{2}=L_{-1}, \quad J_{2} A_{1} J_{2}=A_{-1}, \quad J_{2} A_{2} J_{2}=A_{-2},
$$




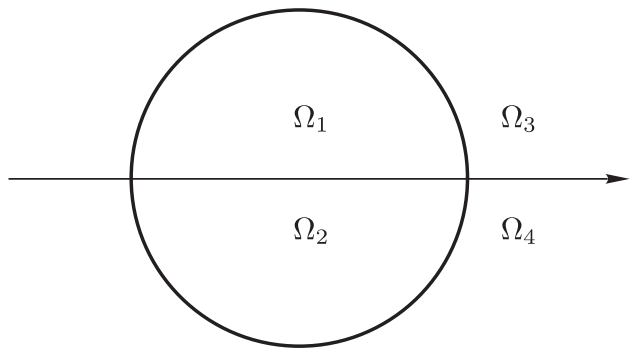

Непрерывный спектр оператора $L$ в случае граничных условий (7б).

где $J_{2}=\operatorname{diag}(1,-1,1)$. Нетрудно проверить, что $|u|^{2}+|v|^{2}$ есть константа, которую мы можем, введя номировку, положить равной единице, $|u|^{2}+|v|^{2}=1$, т. е. вектор $(u, v)$ лежит на трехмерной сфере в $\mathbb{R}^{4}$.

Спектральная теория для оператора Лакса $L(2)$ существенно зависит от граничных условий. У нас есть два естественных способа определить граничные конфигурации, а именно

$$
\begin{array}{ll}
\lim _{x \rightarrow \pm \infty} u(x, t)=1, & \lim _{x \rightarrow \pm \infty} v(x, t)=0, \\
\lim _{x \rightarrow \pm \infty} u(x, t)=0, & \lim _{x \rightarrow \pm \infty} v(x, t)=1 .
\end{array}
$$

Решения Йоста оператора $L$ определяются с использованием асимптотических потенциалов $U_{ \pm, \text {as }}(\lambda)=\lim _{x \rightarrow \pm \infty}\left(\lambda L_{1}+\lambda^{-1} L_{-1}\right)$, которые могут быть приведены к диагональному виду, $g_{0}^{-1} U_{ \pm, \text {as }}(\lambda) g_{0}=J(\lambda)$, для обоих случаев граничных условий: имеем соответственно для (7а) и (7б)

$$
g_{0}=\frac{1}{\sqrt{2}}\left(\begin{array}{ccc}
1 & 0 & 1 \\
1 & 0 & -1 \\
0 & -\sqrt{2} & 0
\end{array}\right), \quad J(\lambda)=\left(\lambda-\lambda^{-1}\right) \operatorname{diag}(1,0,-1)
$$

и

$$
g_{0}=\frac{1}{\sqrt{2}}\left(\begin{array}{ccc}
1 & 0 & -1 \\
0 & \sqrt{2} & 0 \\
1 & 0 & 1
\end{array}\right), \quad J(\lambda)=\left(\lambda+\lambda^{-1}\right) \operatorname{diag}(1,0,-1) .
$$

Непрерывный спектр оператора $L$ для случая (7a) заполняет действительную ось $\mathbb{R}$, в то время как в случае (7б) он является объединением действительной оси и единичной окружности (см. рисунок).

В работе [21] мы построили фундаментальные аналитические решения (ФАР) для $L$ в обоих случаях. Опуская подробности, мы лишь заметим, что эти ФАР могут рассматриваться как решения задач Римана-Гильберта (ЗРГ):

Решения Йоста оператора $L$ и соответствующая им матрица рассеяния $T(\lambda)$ определяются следующим образом:

$$
\lim _{x \rightarrow \pm \infty} \psi_{ \pm}(x, \lambda) e^{-i J(\lambda) x} g_{0}^{-1}=\mathbb{1}, \quad T(\lambda)=\psi_{+}^{-1}(x, \lambda) \psi_{-}(x, \lambda) .
$$

Для случая (7a) ФАР $\chi^{ \pm}$являются решениями следующей ЗРГ:

$$
\chi^{+}(x, \lambda)=\chi^{-}(x, \lambda) G(\lambda), \quad G(\lambda)=\left[S^{-}(\lambda)\right]^{-1} S^{+}(\lambda), \quad \lambda \in \mathbb{R} .
$$


Они удовлетворяют соотношениям симметрии, на которые наложены редукции

$$
\begin{gathered}
\left(\chi^{+}\right)^{\dagger}\left(x, \lambda^{*}\right)=\chi^{-}(x, \lambda), \\
J_{1} \chi^{+}(x,-\lambda) J_{1}=\chi^{-}(x, \lambda), \quad J_{2} \chi^{ \pm}\left(x, \lambda^{-1}\right) J_{2}=\chi^{ \pm}(x, \lambda) .
\end{gathered}
$$

В случае (7б) мы имеем четыре $\Phi А Р ~ \chi^{(j)}(x, \lambda)$, аналитические в областях $\Omega_{j}$ (см. рисунок), которые удовлетворяют следующей ЗРГ на контуре, показанном на рисунке:

$$
\begin{array}{lll}
\chi^{(1)}(x, \lambda)=\chi^{(2)}(x, \lambda)(\lambda), & \lambda \in[-1,1], \\
\chi^{(4)}(x, \lambda)=\chi^{(3)}(x, \lambda) G(\lambda), & & \lambda \in(-\infty,-1] \cup[1, \infty), \\
\chi^{(1)}(x, \lambda)=\chi^{(3)}(x, \lambda) G(\lambda), & \lambda=e^{i \varphi}, \quad \varphi \in(0, \pi), \\
\chi^{(4)}(x, \lambda)=\chi^{(2)}(x, \lambda) G(\lambda), & \lambda=e^{i \varphi}, \quad \varphi \in(\pi, 2 \pi),
\end{array}
$$

где $G(\lambda)=\left(S^{-}(\lambda)\right)^{-1} S^{+}(\lambda)-$ сшивающая функция. ФАР подчиняются следующим соотношениям симметрии:

$$
\begin{array}{cl}
{\left[\chi^{(1)}\left(x, \lambda^{*}\right)\right]^{\dagger}=\left[\chi^{(2)}(x, \lambda)\right]^{-1},} & {\left[\chi^{(4)}\left(x, \lambda^{*}\right)\right]^{\dagger}=\left[\chi^{(3)}(x, \lambda)\right]^{-1},} \\
J_{1} \chi^{(1)}(x,-\lambda) J_{1}=\chi^{(2)}(x, \lambda), & J_{1} \chi^{(4)}(x,-\lambda) J_{1}=\chi^{(3)}(x, \lambda), \\
J_{2} \chi^{(1)}\left(x, \lambda^{-1}\right) J_{2}=\chi^{(4)}(x, \lambda), & J_{2} \chi^{(2)}\left(x, \lambda^{-1}\right) J_{2}=\chi^{(3)}(x, \lambda) .
\end{array}
$$

\section{3. РЕКУРСИОННЫЙ ОПЕРАТОР}

Этот раздел посвящен построению рекурсионного оператора для НЭУ (3). Мы используем метод ГКС, предложенный в работе [17]. Мы рассматриваем рекурсионный оператор как псевдодифференциальный оператор, который отображает симметрию НЭУ в другую симметрию. Основополагающая идея метода ГКС заключается в том, чтобы установить взаимосвязь между двумя ближайшими потоками $\tilde{A}$ и $A$ с эволюционными переменными $\tau$ и $t$ соответственно с помощью равенства [17], [23]

$$
\widetilde{V}=\left(\lambda^{2}+\lambda^{-2}\right) V+B .
$$

Матричнозначные функции $\widetilde{V}$ и $V$ задают вторые операторы $\tilde{A}$ и $A$ пары Лакса следующим образом:

$$
\tilde{A}=i \partial_{\tau}+\tilde{V}(x, t, \lambda), \quad A=i \partial_{t}+V(x, t, \lambda) .
$$

Все приведенные выше величины должны быть инвариантны относительно действия $\mathbb{Z}_{2}$-редукций (5) и (6). Это объясняет выбор коэффициента $\lambda^{2}+\lambda^{-2}$ перед $V$ в соотношении (9), которое является инвариантным относительно замен $\lambda \rightarrow-\lambda$ и $\lambda \rightarrow \lambda^{-1}$. Матрица $B$ выбирается как рациональная функция переменной $\lambda$ :

$$
B=B_{0}+\lambda B_{1}+\frac{1}{\lambda} B_{-1}+\lambda^{2} B_{2}+\frac{1}{\lambda^{2}} B_{-2},
$$

где $B_{-k}=J_{2} B_{k} J_{2}, k=1,2$. Матрицы, входящие в $B$, являются эрмитовыми и имеют блочную структуру:

$$
B_{1} \equiv\left(\begin{array}{cc}
0 & c^{\mathrm{T}} \\
c^{*} & 0
\end{array}\right), \quad B_{2} \equiv\left(\begin{array}{cc}
d & 0 \\
0 & D
\end{array}\right), \quad D \equiv\left(\begin{array}{cc}
\alpha & \beta \\
\beta^{*} & \delta
\end{array}\right) .
$$


В начале нашего рассмотрения заметим, что условие нулевой кривизны операторов Лакса $L$ и $A$ можно записать следующим образом:

$$
i L_{t}=[L, V]
$$

где $L_{t}=\lambda L_{1, t}+\lambda^{-1} L_{-1, t}$. После подстановки (9) в равенство, аналогичное (10), в котором эволюционный параметр $t$ заменяется на $\tau$, мы получаем следующее основное уравнение:

$$
i L_{\tau}=i\left(\lambda^{2}+\frac{1}{\lambda^{2}}\right) L_{t}+[L, B] .
$$

Тогда рекурсионный оператор $\mathcal{R}$ может быть найден из соотношения

$$
\left(\begin{array}{c}
\boldsymbol{u} \\
\boldsymbol{u}^{*}
\end{array}\right)=\mathcal{R}\left(\begin{array}{c}
\boldsymbol{u} \\
\boldsymbol{u}^{*}
\end{array}\right)_{t}
$$

Так как все матрицы являются бесследовыми, мы имеем $d=-\operatorname{tr} D=-(\alpha+\delta)$. Сравнивая коэффициенты перед разными степенями переменной $\lambda$, мы разбиваем уравнение (11) на следующие:

$$
\begin{aligned}
& i L_{1, t}+\left[L_{1}, B_{2}\right]=0 \Rightarrow i \boldsymbol{u}_{t}+\left(D^{*}-d\right) \boldsymbol{u}=0, \\
& i B_{2, x}+\left[L_{1}, B_{1}\right]=0 \Rightarrow i D_{x}+\boldsymbol{u}^{*} \boldsymbol{c}^{\mathrm{T}}-\boldsymbol{c}^{*} \boldsymbol{u}^{\mathrm{T}}=0, \quad i d_{x}+\boldsymbol{u}^{\mathrm{T}} \boldsymbol{c}^{*}-\boldsymbol{c} \boldsymbol{u}^{*}=0, \\
& i L_{1, \tau}=i L_{-1, t}+i B_{1, x}+\left[L_{1}, B_{0}\right]+\left[L_{-1}, B_{2}\right], \\
& i B_{0, x}+\left[L_{1}, B_{-1}\right]+\left[L_{-1}, B_{1}\right]=0 .
\end{aligned}
$$

Остальные соотношения могут быть получены из приведенных выше с помощью умножения каждой величины на $J_{2}$ слева и справа.

Система (12) является линейной относительно элементов матрицы $D$. Ее решение записывается в виде

$$
\begin{aligned}
\alpha & =i\left(u u_{t}^{*}+v^{*} v_{t}\right)-d\left(2|v|^{2}-|u|^{2}\right), \\
\beta & =i\left(v u_{t}^{*}-u^{*} v_{t}\right)+3 d u^{*} v, \\
\delta & =-i\left(u u_{t}^{*}+v^{*} v_{t}\right)-d\left(2|u|^{2}-|v|^{2}\right) .
\end{aligned}
$$

Соотношение

$$
\alpha_{x}|v|^{2}+\delta_{x}|u|^{2}-\beta_{x} u v^{*}-\beta_{x}^{*} u^{*} v=0
$$

является условием разрешимости этой системы. Можно удостовериться в том, что

$$
\boldsymbol{c}=\left(\begin{array}{l}
c \\
s
\end{array}\right)=\frac{i}{2}\left(\begin{array}{c}
u\left(\delta_{x}-\alpha_{x}\right)-2 v \beta_{x}^{*} \\
-v\left(\delta_{x}-\alpha_{x}\right)-2 u \beta_{x}
\end{array}\right)+\varkappa\left(\begin{array}{l}
u \\
v
\end{array}\right)
$$

является общим решением, где $\varkappa$ - произвольная действительная функция, которую следует найти. Решение может быть записано в следующем матричном виде:

$$
\left(\begin{array}{c}
c \\
s \\
c^{*} \\
s^{*}
\end{array}\right)_{x}=\mathcal{A}\left(\begin{array}{c}
\beta \\
\beta^{*} \\
(\alpha-\delta) / 2 \\
(\alpha+\delta) / 2
\end{array}\right)+\varkappa_{x}\left(\begin{array}{l}
u \\
v \\
u^{*} \\
v^{*}
\end{array}\right)+\varkappa\left(\begin{array}{l}
u \\
v \\
u^{*} \\
v^{*}
\end{array}\right)_{x}
$$




$$
\mathcal{A}:=i \frac{d}{d x}\left(\begin{array}{cccc}
0 & -v & -u & 0 \\
-u & 0 & v & 0 \\
v^{*} & 0 & u^{*} & 0 \\
0 & u^{*} & -v^{*} & 0
\end{array}\right) \frac{d}{d x}
$$

Наш следующий шаг заключается в том, что мы ищем функцию $d$, входящую в выражения для $\alpha, \beta$ и $\delta$. Для этого воспользуемся условием (16), которое приводит к линейному дифференциальному уравнению для $d$ :

$$
\begin{aligned}
& 2 d_{x}-i\left(u^{*} u_{t}+v^{*} v_{t}\right)_{x}+i\left[\left(u u_{t}^{*}+v^{*} v_{t}\right)\left(|v|^{2}-|u|^{2}\right)_{x}+\right. \\
& \left.+\left(u v_{t}^{*}-v^{*} u_{t}\right)\left(u^{*} v\right)_{x}-\left(u^{*} v_{t}-v^{*} u_{t}\right)\left(u v^{*}\right)_{x}\right]=0
\end{aligned}
$$

После некоторых простых преобразований мы получаем

$$
d=\frac{i}{2}\left(u u_{t}^{*}+v v_{t}^{*}\right)-\frac{i}{2} \partial_{x}^{-1}\left(u_{t} u_{x}^{*}+v_{t} v_{x}^{*}-u_{t}^{*} u_{x}-v_{t}^{*} v_{x}\right),
$$

где $\partial_{x}^{-1}(\cdot):=\int_{ \pm \infty}^{x}(\cdot) d y$.

Из уравнения (15) непосредственно следует, что выполняется следующее равенство:

$$
i B_{0, x}=2 \operatorname{diag}\left(u c^{*}-u^{*} c+v^{*} s-v s^{*}, u^{*} c-u c^{*}, v s^{*}-v^{*} s\right) .
$$

Учитывая формулы (13), мы выводим, что $B_{0}=\operatorname{diag}(\alpha-\delta,-\alpha, \delta)$. Более того, принимая во внимание структуру уравнения (14), можно легко увидеть, что оператор $\mathcal{R}$ представи́м в следующем виде: $\mathcal{R}=\mathcal{R}_{0}+\mathcal{D}$, где

$$
\begin{aligned}
& \mathcal{R}_{0}=\pi \mathcal{A}\left(\mathcal{B}_{\text {loc }}+\mathcal{B}_{\text {nonl }}\right)+\mathcal{K}, \\
& \mathcal{B}_{\text {loc }}=\frac{i}{4}\left(\begin{array}{cccc}
0 & -4 u^{*} & 2 v\left(3|v|^{2}-1\right) & -6 u^{*} v^{2} \\
2 v^{*}\left(1-3|v|^{2}\right) & 6 u\left(v^{*}\right)^{2} & 0 & 4 u \\
-4 u^{*} & 0 & 3 u\left(|v|^{2}-|u|^{2}\right) & -v\left(1+6|u|^{2}\right) \\
-u^{*} & -v^{*} & 0 & 0
\end{array}\right) \\
& \mathcal{B}_{\text {nonl }}=-\frac{3 i}{4}\left(\begin{array}{c}
2 u^{*} v \\
2 u v^{*} \\
|u|^{2}-|v|^{2} \\
-1 / 3
\end{array}\right) \partial_{x}^{-1}\left[\left(u_{x}^{*}, v_{x}^{*},-u_{x},-v_{x}\right)\right] \\
& \mathcal{K}=-\frac{1}{2}\left(\begin{array}{l}
u \\
v \\
u^{*} \\
v^{*}
\end{array}\right)_{x} \partial_{x}^{-1}\left[\left(u^{*}, v^{*}, u, v\right) \mathcal{A}\left(\mathcal{B}_{\text {loc }}+\mathcal{B}_{\text {nonl }}\right)\right] \\
& \pi=\left(\begin{array}{cccc}
1-|u|^{2} / 2 & -u v^{*} / 2 & -u^{2} / 2 & -u v / 2 \\
-u^{*} v / 2 & 1-|v|^{2} / 2 & -u v / 2 & -v^{2} / 2 \\
-\left(u^{*}\right)^{2} / 2 & -u^{*} v^{*} / 2 & 1-|u|^{2} / 2 & -u^{*} v / 2 \\
-u^{*} v^{*} / 2 & -\left(v^{*}\right)^{2} / 2 & -u v^{*} / 2 & 1-|v|^{2} / 2
\end{array}\right)
\end{aligned}
$$


Второй оператор $\mathcal{D}$ получается из первого и последних двух членов в [14]:

$$
\mathcal{D}=\mathcal{P}_{1}-i \mathcal{P}_{2}\left(\mathcal{B}_{\text {loc }}+\mathcal{B}_{\text {nonl }}\right)+\left(\begin{array}{l}
u \\
v \\
u^{*} \\
v^{*}
\end{array}\right)_{x} \partial_{x}^{-1}\left[\left(u^{*}, v^{*}, u, v\right)\right]
$$

где

$$
\mathcal{P}_{1}=\left(\begin{array}{cccc}
-|v|^{2} & 0 & u^{2} & 0 \\
0 & |u|^{2} & 0 & -v^{2} \\
u^{*, 2} & 0 & -|v|^{2} & 0 \\
o & -v^{*, 2} & 0 & |u|^{2}
\end{array}\right), \quad \mathcal{P}_{2}=\left(\begin{array}{cccc}
u^{2} v^{*} & v|v|^{2} & -7 u & -5 u \\
-u|u|^{2} & u^{*} v^{2} & -7 v & 5 v \\
-v^{*}|v|^{2} & \left(u^{*}\right)^{2} v & 7 u^{*} & 5 u^{*} \\
-u\left(v^{*}\right)^{2} & u^{*}|u|^{2} & 7 v & -5 v
\end{array}\right) .
$$

Фактически $\mathcal{R}_{0}$ представляет собой рекурсионный оператор полиномиального пучка [22]:

$$
L:=i \partial_{x}+\lambda L_{1}, \quad A:=i \partial_{t}+\lambda A_{1}+\lambda^{2} A_{2}
$$

\section{4. РЕКУРСИОННЫЕ ОПЕРАТОРЫ И "КВАДРАТЫ РЕШЕНИЙ"}

Этот подход базируется на соотношениях Вронского, отображающих потенциал оператора Лакса $L$ в данные рассеяния, что позволяет ввести "квадраты решений" для оператора $L$ (см., например, работу [4] и приведенные в ней ссылки).

Соотношения Вронского, выведенные в работе [21], принимают вид

$$
\begin{aligned}
\left.\left\langle i \chi^{-1} J_{0} \chi(x, \lambda)-i J_{0}, E_{\alpha}\right\rangle\right|_{x=-\infty} ^{\infty} & =\int_{-\infty}^{\infty} d x\left\langle\left[L_{1}, J_{0}\right], \Phi_{1}(x, \lambda)\right\rangle, \\
\left.\left\langle\chi^{-1} \delta \chi(x, \lambda), E_{\alpha}\right\rangle\right|_{x=-\infty} ^{\infty} & =-\int_{-\infty}^{\infty} d x\left\langle\delta L_{1}, \Phi_{1}(x, \lambda)\right\rangle,
\end{aligned}
$$

где мы ввели квадраты решений

$$
\Phi_{1}(x, \lambda)=\lambda e_{\alpha}(x, \lambda)+\lambda^{-1} \varphi_{0}\left(e_{\alpha}\right)(x, \lambda)
$$

и

$$
e_{\alpha}(x, \lambda)=\chi E_{\alpha} \chi^{-1}(x, \lambda)
$$

a $E_{\alpha}$ является одним из генераторов Картана-Вейля.

В рамках подхода из работ [4], [6] подбираются определенные "проекции" "квадратов решений" $K_{1 ; \alpha}^{ \pm, \perp}(x, \lambda)$ (см. уравнения $(21)$ ниже), которые вносят вклад в правые части равенств (18). После этого рекурсионные операторы могут быть введены как операторы, которые имеют $K_{1 ; \alpha}^{ \pm, \perp}(x, \lambda)$ в качестве собственных функций.

Введем матрицы

$$
J_{1}=\operatorname{diag}(1,-1,-1), \quad K_{1}=\operatorname{diag}(1,0,-1), \quad K_{0}=\operatorname{diag}(1,-1,1)
$$


и функции

$$
\Phi_{k ; \alpha}^{ \pm}(x, \lambda)=\lambda^{k} e_{\alpha}^{ \pm}(x, \lambda)+\lambda^{-k} \varphi_{0}\left(e_{\alpha}^{ \pm}(x, \lambda)\right)
$$

которые удовлетворяют уравнениям

$$
\begin{gathered}
i \frac{\partial \Phi_{k ; \alpha}}{\partial x}+\left[L_{1}, \Phi_{k+1 ; \alpha}^{ \pm}(x, \lambda)\right]+\left[L_{-1}, \Phi_{k-1 ; \alpha}^{ \pm}(x, \lambda)\right]=0, \\
\varphi_{0}\left(\Phi_{k ; \alpha}^{ \pm}(x, \lambda)\right)=\Phi_{-k ; \alpha}^{ \pm}(x, \lambda), \quad \varphi_{0}\left(\Phi_{0 ; \alpha}^{ \pm}(x, \lambda)\right)=\Phi_{0 ; \alpha}^{ \pm}(x, \lambda), \\
i \frac{\partial \Phi_{0 ; \alpha}}{\partial x}+\left(\mathbb{1}+\varphi_{0}\right)\left[L_{1}, \Phi_{1 ; \alpha}^{ \pm}(x, \lambda)\right]=0 .
\end{gathered}
$$

Кроме того, мы имеем

$$
\left(\lambda+\lambda^{-1}\right) \Phi_{k ; \alpha}^{ \pm}(x, \lambda)=\Phi_{k+1 ; \alpha}^{ \pm}(x, \lambda)+\Phi_{k-1 ; \alpha}^{ \pm}(x, \lambda)
$$

В дальнейшем мы воспользуемся только уравнениями для $\Phi_{0 ; \alpha}^{ \pm}(x, \lambda)$ и $\Phi_{1 ; \alpha}^{ \pm}(x, \lambda)$ :

$$
\begin{gathered}
i \frac{\partial \Phi_{0 ; \alpha}}{\partial x}+\left(\mathbb{1}+\varphi_{0}\right)\left[L_{1}, \Phi_{1 ; \alpha}^{ \pm}(x, \lambda)\right]=0 \\
i \frac{\partial \Phi_{1 ; \alpha}}{\partial x}-\left[L_{1}-L_{-1}, \Phi_{0 ; \alpha}^{ \pm}(x, \lambda)\right]=-\left(\lambda+\lambda^{-1}\right)\left[L_{1}, \Phi_{1 ; \alpha}^{ \pm}(x, \lambda)\right] .
\end{gathered}
$$

Далее, введем разбиения

$$
\Phi_{0 ; \alpha}^{ \pm}(x, \lambda)=H_{0 ; \alpha}^{ \pm}(x, \lambda)+K_{0 ; \alpha}^{ \pm}(x, \lambda), \quad \Phi_{1 ; \alpha}^{ \pm}(x, \lambda)=H_{1 ; \alpha}^{ \pm}(x, \lambda)+K_{1 ; \alpha}^{ \pm}(x, \lambda),
$$

где

$$
H_{0 ; \alpha}^{ \pm}(x, \lambda)=\varphi_{0}\left(H_{0 ; \alpha}^{ \pm}(x, \lambda)\right), \quad K_{0 ; \alpha}^{ \pm}(x, \lambda)=\varphi_{0}\left(K_{0 ; \alpha}^{ \pm}(x, \lambda)\right),
$$

и получим уравнения

$$
\begin{gathered}
i \frac{\partial H_{0 ; \alpha}^{ \pm}}{\partial x}+\left(\mathbb{1}+\varphi_{0}\right)\left[L_{1}, K_{1 ; \alpha}^{ \pm}(x, \lambda)\right]=0, \\
i \frac{\partial K_{0 ; \alpha}^{ \pm}}{\partial x}+\left(\mathbb{1}+\varphi_{0}\right)\left[L_{1}, H_{1 ; \alpha}^{ \pm}(x, \lambda)\right]=0, \\
i \frac{\partial H_{1 ; \alpha}^{ \pm}}{\partial x}-\left[L_{1}-L_{-1}, K_{0 ; \alpha}^{ \pm}(x, \lambda)\right]=-\left(\lambda+\lambda^{-1}\right)\left[L_{1}, K_{1 ; \alpha}^{ \pm}(x, \lambda)\right], \\
i \frac{\partial K_{1 ; \alpha}^{ \pm}}{\partial x}-\left[L_{1}-L_{-1}, H_{0 ; \alpha}^{ \pm}(x, \lambda)\right]=-\left(\lambda+\lambda^{-1}\right)\left[L_{1}, H_{1 ; \alpha}^{ \pm}(x, \lambda)\right] .
\end{gathered}
$$

Интегрирование первых двух уравнений дает

$$
\begin{aligned}
& H_{0 ; \alpha}^{ \pm}(x, \lambda)=h_{00 ; \alpha}^{ \pm}+i\left(\mathbb{1}+\varphi_{0}\right) \partial_{x}^{-1}\left[L_{1}, K_{1 ; \alpha}^{ \pm, \perp}(x, \lambda)\right] \\
& K_{0 ; \alpha}^{ \pm}(x, \lambda)=k_{00 ; \alpha}^{ \pm}+i\left(\mathbb{1}+\varphi_{0}\right) \partial_{x}^{-1}\left[L_{1}, H_{1 ; \alpha}^{ \pm, \perp}(x, \lambda)\right]
\end{aligned}
$$


где $h_{00 ; \alpha}^{ \pm}$и $k_{00 ; \alpha}^{ \pm}$являются матричнозначными константами. Таким образом, мы выразили $K_{0 ; \alpha}^{ \pm}$и $H_{0 ; \alpha}^{ \pm}$через $K_{1 ; \alpha}^{ \pm, \perp}$ и $H_{1 ; \alpha}^{ \pm, \perp}$, где

$$
\begin{aligned}
& H_{1 ; \alpha}^{ \pm, \perp}(x, \lambda)=H_{1 ; \alpha}^{ \pm}(x, \lambda)-\frac{3}{2} L_{2}\left\langle H_{1 ; \alpha}^{ \pm}, L_{2}\right\rangle, \\
& K_{1 ; \alpha}^{ \pm, \perp}(x, \lambda)=K_{1 ; \alpha}^{ \pm}(x, \lambda)-\frac{1}{2} L_{1}\left\langle K_{1 ; \alpha}^{ \pm}, L_{1}\right\rangle
\end{aligned}
$$

и $L_{2}=l_{1}^{2}-(2 / 3) \mathbb{1}$. Теперь мы выведем уравнения для $K_{1 ; \alpha}^{ \pm, \perp}$ и $H_{1 ; \alpha}^{ \pm, \perp}$. Для этого мы подставим выражения (21) в два последних уравнения в (20). Таким образом, мы получаем

$$
\begin{aligned}
i \frac{\partial H_{1 ; \alpha}^{ \pm, \perp}}{\partial x}+ & \frac{3 i}{2} L_{2, x}\left\langle H_{1 ; \alpha}^{ \pm}, L_{2}\right\rangle+\frac{3 i}{2} L_{2} \frac{\partial\left\langle H_{1 ; \alpha}^{ \pm}, L_{2}\right\rangle}{\partial x}-\left[L_{1}-L_{-1}, K_{0 ; \alpha}^{ \pm}(x, \lambda)\right]= \\
& =-\left(\lambda+\lambda^{-1}\right)\left[L_{1}, K_{1 ; \alpha}^{ \pm}(x, \lambda)\right], \\
i \frac{\partial K_{1 ; \alpha}^{ \pm, \perp}}{\partial x}+ & \frac{i}{2} L_{1, x}\left\langle K_{1 ; \alpha}^{ \pm}, L_{1}\right\rangle+\frac{i}{2} L_{1} \frac{\partial\left\langle K_{1 ; \alpha}^{ \pm}, L_{1}\right\rangle}{\partial x}-\left[L_{1}-L_{-1}, H_{0 ; \alpha}^{ \pm}(x, \lambda)\right]= \\
& =-\left(\lambda+\lambda^{-1}\right)\left[L_{1}, H_{1 ; \alpha}^{ \pm}(x, \lambda)\right] .
\end{aligned}
$$

Разобьем левые части этих уравнений на слагаемые, коммутирующие с операторами $L_{1}$ и $L_{2}$, и слагаемые, “ортогональные" им. Чтобы найти коэффициенты $\left\langle H_{1 ; \alpha}^{ \pm}, L_{2}\right\rangle$ и $\left\langle K_{1 ; \alpha}^{ \pm}, L_{1}\right\rangle$, мы умножим обе части первого и второго из уравнений $(22)$ на $L_{2}$ и соответственно на $L_{1}$, после чего вычислим след. В результате получаем

$$
\begin{aligned}
& i\left\langle\frac{\partial H_{1 ; \alpha}^{ \pm, \perp}}{\partial x}, L_{2}\right\rangle+i \frac{\partial\left\langle H_{1 ; \alpha}^{ \pm}, L_{2}\right\rangle}{\partial x}-\left\langle\left[L_{1}-L_{-1}, K_{0 ; \alpha}^{ \pm}(x, \lambda)\right], L_{2}\right\rangle=0 \\
& i\left\langle\frac{\partial K_{1 ; \alpha}^{ \pm, \perp}}{\partial x}, L_{1}\right\rangle+i \frac{\partial\left\langle K_{1 ; \alpha}^{ \pm}, L_{1}\right\rangle}{\partial x}-\left\langle\left[L_{1}-L_{-1}, H_{0 ; \alpha}^{ \pm}(x, \lambda)\right], L_{1}\right\rangle=0 .
\end{aligned}
$$

Интегрируя эти уравнения, имеем

$$
\begin{aligned}
& \left\langle H_{1 ; \alpha}^{ \pm}, L_{2}\right\rangle=h_{01 ; \alpha}^{ \pm}-\partial_{x}^{-1}\left\langle\frac{\partial H_{1 ; \alpha}^{ \pm, \perp}}{\partial x}, L_{2}\right\rangle-i \partial_{x}^{-1}\left\langle\left[L_{1}-L_{-1}, K_{0 ; \alpha}^{ \pm}(x, \lambda)\right], L_{2}\right\rangle \\
& \left\langle K_{1 ; \alpha}^{ \pm}, L_{1}\right\rangle=k_{01 ; \alpha}^{ \pm}-\partial_{x}^{-1}\left\langle\frac{\partial K_{1 ; \alpha}^{ \pm, \perp}}{\partial x}, L_{1}\right\rangle
\end{aligned}
$$

где $k_{01 ; \alpha}^{ \pm}$и $h_{01 ; \alpha}^{ \pm}$являются матричнозначными константами интегрирования.

Опуская подробности, мы получаем

$$
\begin{aligned}
\Lambda_{1} K_{1 ; \alpha}^{ \pm, \perp}= & \left(\lambda+\lambda^{-1}\right) H_{1 ; \alpha}^{ \pm, \perp}- \\
& -\operatorname{ad}_{L_{1}}^{-1}\left(\left[L_{1}-L_{-1}, h_{00}\right]+\frac{3}{2} L_{2, x} \partial_{x}^{-1}\left\langle\left[L_{1}, L_{-1}\right], k_{00}\right\rangle\right) \\
\Lambda_{2} H_{1 ; \alpha}^{ \pm, \perp}= & \left(\lambda+\lambda^{-1}\right) K_{1 ; \alpha}^{ \pm, \perp}+ \\
& +\frac{3}{2} \operatorname{ad}_{L_{1}}^{-1}\left(L_{2, x} h_{01}+i L_{2, x} \partial_{x}^{-1}\left\langle\left[L_{2}, L_{-1}\right], k_{00}\right\rangle\right)-\operatorname{ad}_{L_{1}}^{-1}\left(\left[L_{1}-L_{-1}, k_{00}\right]\right),
\end{aligned}
$$


где $h_{00}$ и $k_{00}$ - произвольные матричнозначные константы, которые мы будем полагать равными нулю. Операторы $\Lambda_{j}, j=1,2$, определяются следующим образом:

$$
\begin{aligned}
\Lambda_{1} K_{1 ; \alpha}^{ \pm, \perp}= & -i \operatorname{ad}_{L_{1}}^{-1}\left(\frac{\partial K_{1 ; \alpha}^{ \pm, \perp}}{\partial x}-\left(\mathbb{1}-\varphi_{0}\right)\left[L_{1}-L_{-1}, \partial_{x}^{-1}\left[L_{1}, K_{1 ; \alpha}^{ \pm, \perp}\right]\right]\right)+ \\
& +\frac{i}{2} \operatorname{ad}_{L_{1}}^{-1} L_{1, x} \partial_{x}^{-1}\left\langle\frac{\partial K_{1 ; \alpha}^{ \pm, \perp}}{\partial x}, L_{1}\right\rangle, \\
\Lambda_{2} H_{1 ; \alpha}^{ \pm, \perp}= & -i \operatorname{ad}_{L_{1}}^{-1}\left(\frac{\partial H_{1 ; \alpha}^{ \pm, \perp}}{\partial x}-\left(\mathbb{1}-\varphi_{0}\right)\left[L_{1}-L_{-1}, \partial_{x}^{-1}\left[L_{1}, H_{1 ; \alpha}^{ \pm, \perp}\right]\right]-\right. \\
& \left.-\frac{3}{2} L_{2, x} \partial_{x}^{-1}\left(\left\langle\frac{\partial H_{1 ; \alpha}^{ \pm, \perp}}{\partial x}, L_{2}\right\rangle+\left\langle\left[L_{2}-L_{-2}, L_{1}-L_{-1}\right], \partial_{x}^{-1}\left[L_{1}, H_{1 ; \alpha}^{ \pm, \perp}\right]\right\rangle\right)\right) .
\end{aligned}
$$

Таким образом, мы получаем

$$
\Lambda_{2} \Lambda_{1} K_{1 ; \alpha}^{ \pm, \perp}=\left(\lambda+\lambda^{-1}\right)^{2} K_{1 ; \alpha}^{ \pm, \perp}, \quad \Lambda_{1} \Lambda_{2} H_{1 ; \alpha}^{ \pm, \perp}=\left(\lambda+\lambda^{-1}\right)^{2} H_{1 ; \alpha}^{ \pm, \perp} .
$$

\section{5. ЗАКЛЮЧЕНИЕ}

Мы получили рекурсионные операторы $\Lambda$ для системы (3), связанной с симметричным пространством $S U(3) / S(U(1) \times U(2))$ с дополнительной $\mathbb{Z}_{2}$-редукцией. Первый способ построения $\Lambda$ опирается на метод ГКС [17], а другой заключается в использовании соотношений Вронского. Они позволяют определить “квадраты решений" оператора $L$, которые рассматриваются как собственные функции рекурсионного оператора $\Lambda$. Наши результаты можно распространить на операторы $L$, связанные с общим симметричным пространством типа A.III $\cong S U(n+k) / S(U(n) \times U(k))$ и с другими типами симметричных пространств.

\section{ПРИЛОЖЕНИЕ}

Здесь мы приводим некоторые промежуточные результаты, используемые в разделе 4. Инволюции $J$ индуцируют $\mathbb{Z}_{2}$-градуировку в алгебре Ли $\mathfrak{g}$ следующим образом: $\mathfrak{g} \equiv \mathfrak{g}^{(0)} \oplus \mathfrak{g}^{(1)}$, где

$$
\begin{aligned}
\mathfrak{g}^{(0)} \equiv\left\{Y: Y=J Y J^{-1}\right\}, & \mathfrak{g}^{(1)} \equiv\left\{X: X=-J X J^{-1}\right\}, \\
Y=\left(\begin{array}{ccc}
-k-n & 0 & 0 \\
0 & k & m \\
0 & m^{*} & n
\end{array}\right), & X=\left(\begin{array}{ccc}
0 & a & b \\
a^{*} & 0 & 0 \\
b^{*} & 0 & 0
\end{array}\right) .
\end{aligned}
$$

Внутренний автоморфизм $\varphi_{0}$ в формуле для “уточненных квадратов решений” (19) может быть представлен в виде $\varphi_{0}(Z) \equiv K_{0} Z K_{0}^{-1}$, где

$$
\begin{array}{lll}
Y=Y^{+}+Y^{-}, & Y^{+}=\left(\begin{array}{ccc}
-k-n & 0 & 0 \\
0 & k & 0 \\
0 & 0 & n
\end{array}\right), & Y^{-}=\left(\begin{array}{ccc}
0 & 0 & 0 \\
0 & 0 & m \\
0 & m^{*} & 0
\end{array}\right), \\
X=X^{+}+X^{-}, & X^{+}=\left(\begin{array}{ccc}
0 & 0 & b \\
0 & 0 & 0 \\
b^{*} & 0 & 0
\end{array}\right), & X^{-}=\left(\begin{array}{ccc}
0 & a & 0 \\
a^{*} & 0 & 0 \\
0 & 0 & 0
\end{array}\right) .
\end{array}
$$


Учтем, что $\varphi_{0}\left(H_{0}\right)=H_{0}$ и $\varphi_{0}\left(K_{0}\right)=K_{0}$, следовательно,

$$
\begin{aligned}
& \left\langle\left[L_{1}-L_{-1}, K_{0 ; \alpha}^{ \pm}(x, \lambda)\right], L_{2}\right\rangle=2|u|^{2}\left(\kappa_{0 ; \alpha}^{ \pm} v^{*}-\kappa_{0 ; \alpha}^{ \pm, *} v\right), \\
& \left\langle\left[L_{1}-L_{-1}, H_{0 ; \alpha}^{ \pm}(x, \lambda)\right], L_{1}\right\rangle=0,
\end{aligned}
$$

где

$$
K_{0 ; \alpha}^{ \pm}=\left(\begin{array}{ccc}
0 & 0 & \kappa_{0 ; \alpha}^{ \pm} \\
0 & 0 & 0 \\
\kappa_{0 ; \alpha}^{ \pm, *} & 0 & 0
\end{array}\right), \quad H_{0 ; \alpha}^{ \pm}=\left(\begin{array}{ccc}
-k_{0 ; \alpha}^{ \pm}-n_{0 ; \alpha}^{ \pm} & 0 & 0 \\
0 & k_{0 ; \alpha}^{ \pm} & 0 \\
0 & 0 & n_{0 ; \alpha}^{ \pm}
\end{array}\right)
$$

Введем обозначения

$$
K_{1 ; \alpha}^{ \pm}=\left(\begin{array}{ccc}
0 & w_{1 ; \alpha}^{ \pm} & z_{1 ; \alpha}^{ \pm} \\
w_{1 ; \alpha}^{ \pm, *} & 0 & 0 \\
z_{1 ; \alpha}^{ \pm, *} & 0 & 0
\end{array}\right), \quad H_{1 ; \alpha}^{ \pm}=\left(\begin{array}{ccc}
-k_{1 ; \alpha}^{ \pm}-n_{1 ; \alpha}^{ \pm} & 0 & 0 \\
0 & k_{1 ; \alpha}^{ \pm} & m_{1 ; \alpha}^{ \pm} \\
0 & m_{1 ; \alpha}^{ \pm, *} & n_{1 ; \alpha}^{ \pm}
\end{array}\right)
$$

Тогда

$$
\begin{aligned}
\left\langle H_{1 ; \alpha}^{ \pm}, L_{2}\right\rangle= & h_{01 ; \alpha}^{ \pm}-\partial_{x}^{-1}\left\langle\frac{\partial H_{1 ; \alpha}^{ \pm, \perp}}{\partial x}, L_{2}\right\rangle-2 i \partial_{x}^{-1}|u|^{2}\left(v^{*} k_{00 ; \alpha}^{ \pm}-v k_{00 ; \alpha}^{ \pm, *}\right)+ \\
& +8 \partial_{x}^{-1}|u|^{2}\left(v^{*} \partial_{x}^{-1}\left(u m_{1 ; \alpha}^{ \pm}\right)+v \partial_{x}^{-1}\left(u^{*} m_{1 ; \alpha}^{ \pm, *}\right)\right), \\
\left\langle K_{1 ; \alpha}^{ \pm}, L_{1}\right\rangle= & k_{01 ; \alpha}^{ \pm}-\partial_{x}^{-1}\left\langle\frac{\partial K_{1 ; \alpha}^{ \pm, \perp}}{\partial x}, L_{1}\right\rangle .
\end{aligned}
$$

Благодарности. Авторы благодарят Royal Society и Bulgarian Academy of Sciences за поддержку в рамках совместного исследовательского проекта "Reductions of Nonlinear Evolution Equations and Analytical Spectral Theory". В. С. Герджиков и Г. Г. Граховский также благодарны организаторам конференции "Нелинейная физика: теория и эксперимент. VI" за финансовую поддержку и теплый прием в Галлиполи. Работа Г. Г. Граховского была поддержана Science Foundation of Ireland (грант № 09/RFP/MTH2144).

\section{Список литературы}

[1] В. Г. Дринфельд, В. В. Соколов, “Алгебры Ли и уравнения типа Кортевега-де Фриза”, Итоги науки и техн. Сер. Соврем. пробл. мат. Нов. достиж., 24, ред. Р. В. Гамкрелидзе, ВИНИТИ, М., 1984, 81-180.

[2] В.Е. Захаров, С.В. Манаков, С.П. Новиков, Л.П. Питаевский, Теория солитонов: Метод обратной задачи, Наука, М., 1980.

[3] Л. А. Тахтаджян, Л. Д. Фаддеев, Гамильтонов подход в теории солитонов, Наука, М., 1986.

[4] V.S. Gerdjikov, G. Vilasi, A. B. Yanovski, Integrable Hamiltonian Hierarchies. Spectral and Geometric Methods, Lecture Notes in Phys., 748, Springer, Berlin, 2008.

[5] M. J. Ablowitz, D. J. Kaup, A. C. Newell, H. Segur, Stud. Appl. Math., 53:4 (1974), 249-315.

[6] D. J. Kaup, A. C. Newell, J. Math. Phys., 19:4 (1978), 798-801.

[7] V.S. Gerdžikov, E. H. Hristov, Bulgar. J. Phys., 7:2 (1980), 119-133.

[8] V.S. Gerdjikov, M. I. Ivanov, Bulgar. J. Phys., 10:2 (1983), 130-143.

[9] А. В. Михайлов, Писъма в ЖЭТФ, 30:7 (1979), 443-448. 
[10] А. В. Михайлов, Писъма в ЖЖЭТФ, 32:2 (1980), 187-192.

[11] A. V. Mikhailov, Physica D, 3:1-2 (1981), 73-117.

[12] A. V. Mikhailov, Phys. Lett. A, 92:2 (1982), 51-55.

[13] A. V. Mikhailov, M. A. Olshanetsky, A. M. Perelomov, Comm. Math. Phys., 79:4 (1981), 473-488.

[14] S. Lombardo, J. A. Sanders, Comm. Math. Phys., 299:3 (2010), 793-824, arXiv: 0912.1697.

[15] S. Lombardo, A. V. Mikhailov, J. Phys. A, 37:31 (2004), 7727-7742, arXiv: nlin/0404013.

[16] S. Lombardo, A. Mikhailov, Comm. Math. Phys., 258:1 (2005), 179-202, arXiv: math-ph/0407048.

[17] M. Gürses, A. Karasu, V. V. Sokolov, J. Math. Phys., 40:12 (1999), 6473-6490, arXiv: solv-int/9909003.

[18] И. Ц. Голубчик, В. В. Соколов, ТМФ, 124:1 (2000), 62-71.

[19] В. Е. Адлер, А. Б. Шабат, Р. И. Ямилов, ТМФ, 125:3 (2000), 355-424.

[20] A. V. Mikhailov, V. V. Sokolov, "Symmetries of differential equations and the problem of integrability", Integrability, Lecture Notes in Phys., 767, ed. A. V. Mikhailov, Springer, Berlin, 2009, 19-88.

[21] V. Gerdjikov, A. V. Mikhailov, T. I. Valchev, J. Phys. A, 43:43 (2010), 434015, 13 pp.

[22] V.S. Gerdjikov, A. V. Mikhailov, T. I. Valchev, J. Geom. Symmetry Phys., 20 (2010), 1-34.

[23] J. P. Wang, J. Math. Phys., 50:2 (2009), 023506, 25 pp., arXiv: 0809.3899.

[24] S. Helgason, Differential Geometry, Lie Groups and Symmetric Spaces, Pure Appl. Math., 80, Academic Press, New York, 1978. 